\title{
TU/e EmonOWEN

\section{Synthesis of trajectory-dependent control Lyapunov functions by a single linear program}

\section{Citation for published version (APA):}

Lazar, M., \& Jokic, A. (2009). Synthesis of trajectory-dependent control Lyapunov functions by a single linear program. In R. Majumdar, \& P. Tabuada (Eds.), Proceedings 12th international conference on Hybrid systems : computation and control, HSCC 2009, April 13-15, 2009, San Francisco, California (pp. 237-251). (Lecture Notes in Computer Science; Vol. 5469). Springer. https://doi.org/10.1007/978-3-642-00602-9_17

DOI:

10.1007/978-3-642-00602-9_17

Document status and date:

Published: 01/01/2009

\section{Document Version:}

Publisher's PDF, also known as Version of Record (includes final page, issue and volume numbers)

\section{Please check the document version of this publication:}

- A submitted manuscript is the version of the article upon submission and before peer-review. There can be important differences between the submitted version and the official published version of record. People interested in the research are advised to contact the author for the final version of the publication, or visit the $\mathrm{DOI}$ to the publisher's website.

- The final author version and the galley proof are versions of the publication after peer review.

- The final published version features the final layout of the paper including the volume, issue and page numbers.

Link to publication

\section{General rights}

Copyright and moral rights for the publications made accessible in the public portal are retained by the authors and/or other copyright owners and it is a condition of accessing publications that users recognise and abide by the legal requirements associated with these rights.

- Users may download and print one copy of any publication from the public portal for the purpose of private study or research.

- You may not further distribute the material or use it for any profit-making activity or commercial gain

- You may freely distribute the URL identifying the publication in the public portal.

If the publication is distributed under the terms of Article 25fa of the Dutch Copyright Act, indicated by the "Taverne" license above, please follow below link for the End User Agreement:

www.tue.nl/taverne

Take down policy

If you believe that this document breaches copyright please contact us at:

openaccess@tue.nl

providing details and we will investigate your claim. 


\title{
Synthesis of Trajectory-Dependent Control Lyapunov Functions by a Single Linear Program
}

\author{
Mircea Lazar and Andrej Jokic \\ Dept. of Electrical Eng., Eindhoven Univ. of Technology, \\ P.O. Box 513, 5600 MB Eindhoven, The Netherlands \\ m.lazar@tue.nl, a.jokic@tue.nl
}

\begin{abstract}
Although control Lyapunov functions (CLFs) provide a mature framework for the synthesis of stabilizing controllers, their application in the field of hybrid systems remains scarce. One of the reasons for this is conservativeness of Lyapunov conditions. This article proposes a methodology that reduces conservatism of CLF design and is applicable to a wide class of discrete-time nonlinear hybrid systems. Rather than searching for global CLFs off-line, we focus on synthesizing CLFs by solving on-line an optimization problem. This approach makes it possible to derive a trajectory-dependent $C L F$, which is allowed to be locally non-monotone. Besides the theoretical appeal of the proposed idea, we indicate that for systems affine in control and CLFs based on infinity norms, the corresponding on-line optimization problem can be formulated as a single linear program.
\end{abstract}

\section{Introduction}

Control Lyapunov functions (CLFs) [1,2] represent perhaps the most popular tool for synthesizing control laws that achieve stability. The interested reader is referred to the surveys [3,4] for a complete historical account. The classical approach for smooth continuous-time systems is based on the design of an explicit feedback law off-line, which renders the derivative of a candidate CLF negative. Conditions under which these results can be extended to sampled-data nonlinear systems using their approximate discrete-time models can be found in [5]. An important article on control Lyapunov functions for discrete-time systems is 6 . Therein, classical continuous-time results regarding existence of smooth CLFs are reproduced for the discrete-time case.

Despite the popularity of CLFs within smooth nonlinear systems theory, there is still a significant gap in the usage of CLFs in stabilization of hybrid systems. One of the reasons for this is conservativeness of the sufficient conditions for Lyapunov asymptotic stability [7,8], which are employed by most methods for constructing CLFs. This makes classical CLFs overconservative for discontinuous nonlinear and hybrid systems, as observed in the seminal paper 9]. Ever since, the focus has been on designing less conservative types of Lyapunov functions for specific relevant classes of hybrid systems. For example, piecewise quadratic (PWQ) functions were exploited in stability analysis and synthesis

R. Majumdar and P. Tabuada (Eds.): HSCC 2009, LNCS 5469, pp. 237-251, 2009.

(C) Springer-Verlag Berlin Heidelberg 2009 
problems for continuous-time and discrete-time piecewise affine (PWA) systems in [10, 11], 12. Further relaxations were proposed in 13] for discrete-time switched linear systems, using parameter dependent PWQ Lyapunov functions. More recently, a hybrid CLF (which combines two different CLFs) was employed in 14 to stabilize hybrid systems with discrete dynamics (e.g., hybrid systems with discrete states and/or inputs).

To summarize, to some extent, the state-of-the-art methods for stability analysis of discrete-time hybrid systems (mostly PWA and switched linear systems) rely on the off-line search for globally defined PWQ Lyapunov functions. One of the most significant relaxations is that each quadratic function, which is part of the PWQ global function, is required to be positive definite and/or satisfy decreasing conditions only in a subset of the state-space, relaxation often referred to as the $\mathcal{S}$-procedure [10]. From a numerical point of view, the existing tools require solving a semidefinite programming problem. However, when it comes to synthesis of CLFs, which consists of simultaneously searching for a PWQ Lyapunov function and a state-feedback control law, the $\mathcal{S}$-procedure leads to a nonlinear matrix inequality that has not been solved systematically so far, although serious efforts have been put in this direction.

Next, we present a motivating example which suffers from this drawback.

\subsection{Motivating Example}

Consider the following piecewise linear (PWL) system from [], Chapter 3:

$$
x(k+1)=A_{j} x(k)+B u(k) \quad \text { if } \quad E_{j} x(k) \geq 0, \quad k \in \mathbb{Z}_{+},
$$

where $j=\{1,2,3,4\}$,

$$
A_{1}=\left[\begin{array}{cc}
0.5 & 0.61 \\
0.9 & 1.345
\end{array}\right], A_{2}=\left[\begin{array}{rr}
-0.92 & 0.644 \\
0.758 & -0.71
\end{array}\right], B=\left[\begin{array}{l}
1 \\
0
\end{array}\right], A_{3}=A_{1}, A_{4}=A_{2} .
$$

The partitioning of the state-space is given by

$$
E_{1}=-E_{3}=\left[\begin{array}{cc}
-1 & 1 \\
-1 & -1
\end{array}\right], E_{2}=-E_{4}=\left[\begin{array}{cc}
-1 & 1 \\
1 & 1
\end{array}\right]
$$

As shown in [8] the synthesis problem 1 for this system in closed-loop with a PWL state-feedback law is not feasible for a common quadratic or a PWQ Lyapunov function without the $\mathcal{S}$-procedure relaxation. However, a solution to the synthesis problem for a PWQ Lyapunov function with the $\mathcal{S}$-procedure has been found in [8] at the expense of a significant computational complexity (i.e. a griding approach was used to solve a bilinear matrix inequality).

This indicates that there are even very simple classes of discrete-time hybrid systems for which a systematic and efficient synthesis method based on CLFs is not available.

${ }^{1}$ Notice that the above example is a "flower system" for the synthesis problem, similarly as the example introduced in [10] is a "flower system" for the analysis problem. 
Remark 1. Existing on-line optimization based controllers, such as model predictive control algorithms, make use of the above-mentioned off-line synthesis methods to obtain an a priori stability guarantee, see, for example, [15, 16, Hence, they are also affected by the limitations of these methods.

Motivated by the above example, in this paper we propose a new methodology that reduces significantly the conservatism of CLF design for discrete-time systems. Rather than searching for global CLFs off-line, we focus on synthesizing time-variant CLFs by solving on-line an optimization problem. As such, trajectory-dependent CLFs that are allowed to be locally non-monotone can be derived. This approach offers the "least conservative" relaxation possible, in the sense that for a CLF with a fixed structure that incorporates some time-variant parameters, a possibly different value of these parameters is assigned to each measured state. Furthermore, the stabilization conditions that involve the CLF are only imposed along the closed-loop trajectory generated on-line. Numerically, we indicate that for piecewise continuous (PWC) nonlinear systems affine in control and CLFs based on infinity norms, the on-line optimization problem can be formulated as a single linear program. The effectiveness of the developed theory is demonstrated on the motivating example presented above.

\section{Preliminaries}

In this section we recall preliminary notions and fundamental stability results.

\subsection{Basic Notions and Definitions}

Let $\mathbb{R}, \mathbb{R}_{+}, \mathbb{Z}$ and $\mathbb{Z}_{+}$denote the field of real numbers, the set of non-negative reals, the set of integer numbers and the set of non-negative integers, respectively. We use the notation $\mathbb{Z}_{\geq c_{1}}$ and $\mathbb{Z}_{\left(c_{1}, c_{2}\right]}$ to denote the sets $\left\{k \in \mathbb{Z}_{+} \mid k \geq c_{1}\right\}$ and $\left\{k \in \mathbb{Z}_{+} \mid c_{1}<k \leq c_{2}\right\}$, respectively, for some $c_{1}, c_{2} \in \mathbb{Z}_{+}$. For a set $\mathcal{S} \subseteq \mathbb{R}^{n}$, we denote by $\operatorname{int}(\mathcal{S})$ the interior and by $\operatorname{cl}(\mathcal{S})$ the closure of $\mathcal{S}$. A polyhedron (or a polyhedral set) in $\mathbb{R}^{n}$ is a set obtained as the intersection of a finite number of open and/or closed half-spaces. For a vector $x \in \mathbb{R}^{n},[x]_{i}$ denotes the $i$-th element of $x$. A vector $x \in \mathbb{R}^{n}$ is said to be nonnegative (nonpositive) if $[x]_{i} \geq 0$ $\left([x]_{i} \leq 0\right)$ for all $i \in\{1, \ldots n\}$, and in that case we write $x \geq 0(x \leq 0)$. For a vector $x \in \mathbb{R}^{n}$ let $\|\cdot\|$ denote an arbitrary $p$-norm. Let $\|x\|_{\infty}:=\max _{i=1, \ldots, n}\left|[x]_{i}\right|$, where $|\cdot|$ denotes the absolute value. In the Euclidean space $\mathbb{R}^{n}$ the standard inner product is denoted by $\langle\cdot, \cdot\rangle$ and the associated norm is denoted by $\|\cdot\|_{2}$, i.e. for $x \in \mathbb{R}^{n},\|x\|_{2}=\langle x, x\rangle^{\frac{1}{2}}=\left(x^{\top} x\right)^{\frac{1}{2}}$. For a matrix $Z \in \mathbb{R}^{m \times n},[Z]_{i j}$ denotes the element in the $i$-th row and $j$-th column of $Z$. Given $Z \in \mathbb{R}^{m \times n}$ and $I \subseteq\{1, \ldots, m\}$, we write $[Z]_{I}$ to denote a submatrix of $Z$ formed by rows $I$ of $Z$. For a matrix $Z \in \mathbb{R}^{m \times n}$ let $\|Z\|:=\sup _{x \neq 0} \frac{\|Z x\|}{\|x\|}$ denote its corresponding induced matrix norm. It is well known that $\|Z\|_{\infty}=\max _{1 \leq i \leq m} \sum_{j=1}^{n}\left|[Z]_{i j}\right|$.

A function $\varphi: \mathbb{R}_{+} \rightarrow \mathbb{R}_{+}$belongs to class $\mathcal{K}$ if it is continuous, strictly increasing and $\varphi(0)=0$. A function $\varphi: \mathbb{R}_{+} \rightarrow \mathbb{R}_{+}$belongs to class $\mathcal{K}_{\infty}$ if $\varphi \in \mathcal{K}$ and it is radially unbounded (i.e. $\lim _{s \rightarrow \infty} \varphi(s)=\infty$ ). A function $\beta: \mathbb{R}_{+} \times \mathbb{R}_{+} \rightarrow \mathbb{R}_{+}$ 
belongs to class $\mathcal{K} \mathcal{L}$ if for each fixed $k \in \mathbb{R}_{+}, \beta(\cdot, k) \in \mathcal{K}$ and for each fixed $s \in \mathbb{R}_{+}, \beta(s, \cdot)$ is decreasing and $\lim _{k \rightarrow \infty} \beta(s, k)=0$.

\subsection{Lyapunov Asymptotic Stability}

Consider the discrete-time autonomous nonlinear system

$$
x(k+1) \in \Phi(x(k)), \quad k \in \mathbb{Z}_{+},
$$

where $x(k) \in \mathbb{R}^{n}$ is the state at the discrete-time instant $k$ and the mapping $\Phi$ : $\mathbb{R}^{n} \hookrightarrow \mathbb{R}^{n}$ is an arbitrary nonlinear, possibly discontinuous, set-valued function. For simplicity, we assume that the origin is an equilibrium of (2), i.e. $\Phi(0)=\{0\}$. The following definitions give a strong characterization of invariance and stability for the difference inclusion (2), in the sense that these properties are required to hold for all possible trajectories generated by (2), and not just for one of them.

Definition 1. We call a set $\mathcal{P} \subseteq \mathbb{R}^{n}$ positively invariant (PI) for system (2) if for all $x \in \mathcal{P}$ it holds that $\Phi(x) \subseteq \mathcal{P}$.

Definition 2. Let $\mathbb{X}$ with $0 \in \operatorname{int}(\mathbb{X})$ be a subset of $\mathbb{R}^{n}$. We call system (2) $A S(\mathbb{X})$ if there exists a $\mathcal{K} \mathcal{L}$-function $\beta(\cdot, \cdot)$ such that, for each $x(0) \in \mathbb{X}$ it holds that all corresponding state trajectories of (2) satisfy $\|x(k)\| \leq \beta(\|x(0)\|, k)$, $\forall k \in \mathbb{Z}_{+}$. We call system (2) globally asymptotically stable if it is $A S\left(\mathbb{R}^{n}\right)$.

Theorem 1. Let $\mathbb{X}$ be a PI set for (2) with $0 \in \operatorname{int}(\mathbb{X})$. Furthermore, let $\alpha_{1}, \alpha_{2} \in$ $\mathcal{K}_{\infty}, \rho \in \mathbb{R}_{[0,1)}$ and let $V: \mathbb{Z}_{+} \times \mathbb{R}^{n} \rightarrow \mathbb{R}_{+}$be a function such that:

$$
\begin{aligned}
\alpha_{1}(\|x\|) \leq V(k, x) & \leq \alpha_{2}(\|x\|), \forall x \in \mathbb{X}, \forall k \in \mathbb{Z}_{+}, \\
\forall x(0) \in \mathbb{X}, \quad V\left(k+1, x^{+}\right) & \leq \rho V(k, x(k))
\end{aligned}
$$

for all $x^{+} \in \Phi(x(k)), k \in \mathbb{Z}_{+}$. Then system (2) is $A S(\mathbb{X})$.

The proof of the above theorem can be obtained mutatis mutandis from the proofs given in [17, 8] by replacing the difference equation with the difference inclusion as in (2). It is worth to point out that if $V(\cdot)$ is a continuous and timeinvariant function, the above theorem can be recovered from Theorem 2.8 of [18], which gives sufficient conditions for robust $\mathcal{K} \mathcal{L}$-stability of difference inclusions. We call a function $V(\cdot, \cdot)$ that satisfies (3) a time-variant Lyapunov function.

\section{Trajectory-Dependent CLFs for Discrete-Time Systems}

Consider the discrete-time constrained nonlinear system

$$
x(k+1)=\phi(x(k), u(k)), \quad k \in \mathbb{Z}_{+},
$$

where $x(k) \in \mathbb{X} \subseteq \mathbb{R}^{n}$ is the state and $u(k) \in \mathbb{U} \subseteq \mathbb{R}^{m}$ is the control input. $\phi: \mathbb{R}^{n} \times \mathbb{R}^{m} \rightarrow \mathbb{R}^{n}$ is an arbitrary nonlinear function, possibly discontinuous, with $\phi(0,0)=0$. We assume that $0 \in \operatorname{int}(\mathbb{X})$ and $0 \in \operatorname{int}(\mathbb{U})$. 
Remark 2. Since we allow $\phi(\cdot, \cdot)$ to be discontinuous, the following fairly wide class of hybrid systems is accounted for; Piecewise continuous nonlinear systems:

$$
x(k+1)=\phi(x(k), u(k)):=\phi_{j}(x(k), u(k)) \text { if } x(k) \in \Omega_{j}, \quad k \in \mathbb{Z}_{+},
$$

where each $\phi_{j}: \Omega_{j} \times \mathbb{U} \rightarrow \mathbb{R}^{n}, j \in \mathcal{S}$, is a continuous, possibly nonlinear function in $x$ and $\mathcal{S}:=\{1,2, \ldots, s\}$ is a finite set of indices. The collection $\left\{\Omega_{j} \subseteq \mathbb{R}^{n} \mid j \in \mathcal{S}\right\}$ defines a partition of $\mathbb{X}$, meaning that $\cup_{j \in \mathcal{S}} \Omega_{j}=\mathbb{X}$ and $\Omega_{i} \cap \Omega_{j}=\emptyset$, with the sets $\Omega_{j}$ not necessarily closed. In most sections of the paper we will omit the explicit reference to the functions $\phi_{j}(\cdot, \cdot)$ and the partition $\left\{\Omega_{j}\right\}_{j \in \mathcal{S}}$ for brevity.

Definition 3. Let $\alpha_{1}, \alpha_{2} \in \mathcal{K}_{\infty}$ and let $\rho \in \mathbb{R}_{[0,1)}$. A function $V: \mathbb{Z}_{+} \times \mathbb{R}^{n} \rightarrow \mathbb{R}_{+}$ that satisfies

$$
\alpha_{1}(\|x\|) \leq V(k, x) \leq \alpha_{2}(\|x\|), \quad \forall x \in \mathbb{X}, \forall k \in \mathbb{Z}_{+}
$$

and for which there exists a control law $u: \mathbb{X} \rightarrow \mathbb{U}$ such that for any $x(0) \in \mathbb{X}$

$$
V(k+1, \phi(x(k), u(x(k)))) \leq \rho V(k, x(k)) \quad \text { for all } k \in \mathbb{Z}_{+}
$$

is called a time-variant control Lyapunov function (tvCLF) in $\mathbb{X}$ for system (4).

Next, based on Definition 3, we formulate the following optimization problem.

Problem 1. Let $\alpha_{1}, \alpha_{2} \in \mathcal{K}_{\infty}, \rho \in \mathbb{R}_{[0,1)}$ and the structure of a candidate tvCLF $V(\cdot, \cdot)$ be fixed such that (6) holds for all $x \in \mathbb{X}$ and all $k \in \mathbb{Z}_{+}$. At time $k \in \mathbb{Z}_{+}$ measure $x(k)$ and calculate $V(k, x(k))$ and a control action $u(k)$ such that:

$$
\begin{aligned}
& u(k) \in \mathbb{U}, \quad \phi(x(k), u(k)) \in \mathbb{X}, \\
& V(k, \phi(x(k), u(k))) \leq \rho V(k, x(k)), \\
& V(k, x(k)) \leq V(k-1, x(k)) \text { if } k \in \mathbb{Z}_{\geq 1} .
\end{aligned}
$$

The reasoning employed to construct the constraints in Problem 1 is graphically depicted in Figure 1, first plot from left to right. Let $\pi(x(k)):=\{u(x(k)) \mid$ $\exists V(k, \cdot)$ s.t. (6) - (7) hold $\}$ and let $\phi_{\mathrm{cl}}(x, \pi(x)):=\{\phi(x, u) \mid u \in \pi(x)\}$. Notice that for a given $x(0) \in \mathbb{X}$, the inequalities (7) generate, besides a sequence of sets of feasible control actions $\{\pi(x(k))\}_{k \in \mathbb{Z}_{+}}$, also a sequence of sets of feasible realizations of a tvCLF, i.e. $\mathcal{V}(V(k-1), x(k)):=\{V(k, \cdot) \mid$ $\exists u(x(k)$ s.t. (6) - (7) hold) $\}$ for any $k \in \mathbb{Z}_{\geq 1}$. Implicitly, $\pi(x(k))$ also depends on $V(k-1, \cdot)$, but we omitted this dependency for brevity of notation. At $k=0$, both $\pi(x(0))$ and $\mathcal{V}(x(0))$ depend on $x(0)$ only and their definition is recovered by removing (7C) in the definitions given above for $k \in \mathbb{Z}_{\geq 1}$.

Theorem 2. Let $\alpha_{1}, \alpha_{2} \in \mathcal{K}_{\infty}$ be given. Suppose that Problem 11 is feasible for all states $x$ in $\mathbb{X}$. Then the difference inclusion

$$
x(k+1) \in \phi_{c l}(x(k), \pi(x(k))), \quad k \in \mathbb{Z}_{+},
$$

is $A S(\mathbb{X})$. 
Proof. Let $x(k) \in \mathbb{X}$ for some $k \in \mathbb{Z}_{+}$. Then, feasibility of Problem 1 ensures that $x(k+1) \in \phi_{\mathrm{cl}}(x(k), \pi(x(k))) \subseteq \mathbb{X}$ due to constraint (7a). Hence, Problem 1 remains feasible and thus, $\mathbb{X}$ is a PI set for system (8). As $V(k, x)$ satisfies (6) for all $x \in \mathbb{X}$ and all $k \in \mathbb{Z}_{+}$by assumption and hence, it satisfies (3a), we only need to show that $V(k, x(k))$ also satisfies inequality (3b) for all $x(0) \in \mathbb{X}$ and all $k \in \mathbb{Z}_{+}$. At time $k=0$, for any $x(0) \in \mathbb{X}$ we have that $V(0, \phi(x(0), u(0))) \leq \rho V(0, x(0))$ for all $u(0) \in \pi(x(0))$. Furthermore, at time $k=1$ it holds that $V(1, x(1)) \leq V(0, x(1))=V(0, \phi(x(0), u(0))) \leq \rho V(0, x(0))$ for all $u(0) \in \pi(x(0))$. Thus, inequality (3b) holds for system (8) for $k=0$ and all $x(0) \in \mathbb{X}$. We will show next that inequality $(3 \mathrm{~b})$ holds for any $k \in \mathbb{Z}_{\geq 1}$. Due to positive invariance of $\mathbb{X}$, for any $x(0) \in \mathbb{X}$ we have that inequality (7b) is feasible at time $k$ and inequality (7c) is feasible at time $k+1$ for any $k \in \mathbb{Z}_{>1}$. Hence,

$$
V(k+1, x(k+1)) \leq V(k, x(k+1))=V(k, \phi(x(k), u(k))) \leq \rho V(k, x(k)),
$$

for all $u(k) \in \pi(x(k)), k \in \mathbb{Z}_{\geq 1}$ and all $x(0) \in \mathbb{X}$. Then, $\operatorname{AS}(\mathbb{X})$ of system (8) follows from Theorem 1 .

Notice that the result of Theorem 2 is of the type "feasibility implies stability" and as such, we have assumed that Problem 1 is feasible for all $x \in \mathbb{X}$. For a given $x(0) \in \mathbb{X}$, by solving Problem 1 on-line in a receding horizon fashion (assuming that it remains feasible at all future instances), one does not obtain a tvCLF in $\mathbb{X}$, but only a tvCLF valid for the corresponding closed-loop state trajectory $\{x(k)\}_{k \in \mathbb{Z}_{+}}$. Therefore, it makes sense to introduce the following formal definition.

Definition 4. Consider Problem 1. For any $x(0) \in \mathbb{X}$ such that the sets $\pi(x(0))$, $\pi(x(k)), \mathcal{V}(x(0))$ and $\mathcal{V}(V(k-1, \cdot), x(k))$ are non-empty for all $k \in \mathbb{Z}_{\geq 1}$, we call a sequence $\{V(k, \cdot)\}_{k \in \mathbb{Z}_{+}}$with $V(0, \cdot) \in \mathcal{V}(x(0)), V(k, \cdot) \in \mathcal{V}(V(k-1, \cdot), x(k))$ for all $k \in \mathbb{Z}_{\geq 1}$ a trajectory-dependent control Lyapunov function (tdCLF).

It is interesting to point out that a trajectory-dependent CLF can be interpreted as an approximation along a particular trajectory of a possibly very complex global time-invariant CLF. The tdCLF concept can also be extended to deal with a set of trajectories that originate from a particular set of initial conditions of interest.

Furthermore, observe that the $\mathcal{S}$-procedure relaxation proposed in [10] for PWA systems is recovered as a particular case of the design presented in this section, i.e. for $V(k, x(k))$ time invariant as long as $x(k) \in \Omega_{j}$ for some $j \in \mathcal{S}$.

In the next subsection we will briefly discuss the possibility of enlarging the feasible domain of Problem 1 considerably.

\subsection{Non-monotone tdCLFs}

The inequalities (7) can be further significantly relaxed by allowing the candidate tdCLF to be locally non-monotone. This can be done by replacing the inequalities (7b) and (7c) with: 

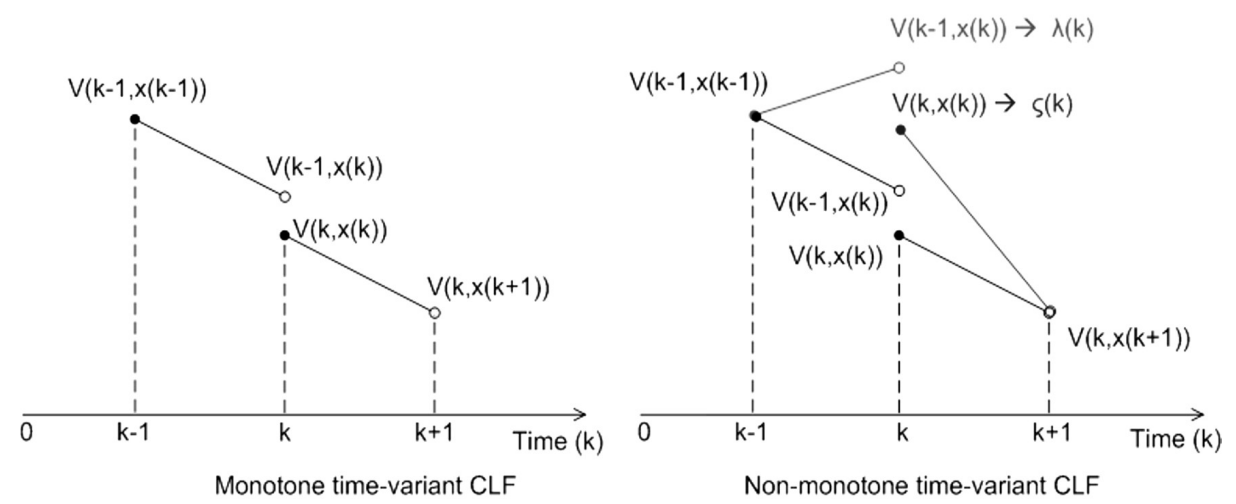

Fig. 1. A graphical illustration of tvCLFs

$$
\begin{aligned}
& V(k, \phi(x(k), u(k))) \leq \rho V(k, x(k))+\lambda(k), \\
& V(k, x(k)) \leq V(k-1, x(k))+\zeta(k) \text { if } k \in \mathbb{Z}_{\geq 1},
\end{aligned}
$$

respectively, where $\lambda(k) \in \mathbb{R}_{+}$and $\zeta(k) \in \mathbb{R}_{+}$are additional variables. For a graphical illustration see Figure 1 the second plot from left to right. Whenever $\lambda(k) \rightarrow 0$ and $\zeta(k) \rightarrow 0$ as $k \rightarrow \infty$ is a priori guaranteed, the closed-loop asymptotic stability result of Theorem 2 still holds. An appealing solution to guarantee this property is to define $\lambda(k)$ and $\zeta(k)$ as outputs of an artificial dynamical system. Then the behavior of $\lambda(k)$ and $\zeta(k)$ can be kept non-monotone, which implies non-monotonicity of $V(\cdot, \cdot)$, while $\lim _{k \rightarrow \infty} \lambda(k)=0$ and $\lim _{k \rightarrow \infty} \zeta(k)=0$ can be ensured through partial stability [19] of the artificial system. The construction of such an artificial system is the object of undergoing research. Alternatively, $\lambda(k) \in \mathbb{R}_{+}$and $\zeta(k) \in \mathbb{R}_{+}$can be set as optimization variables. Then, adding a suitably defined 2 cost function $J(\lambda(k), \zeta(k))$ to Problem 11 and minimizing over $J(\cdot, \cdot)$ for a given $x(k)$ results in optimizing the trade-off between (i) feasibility of Problem 1 and (ii) stabilization.

Remark 3. The relaxation proposed in (9b) recovers as a particular case the one proposed in 9], where it is allowed for the Lyapunov function not to decrease when the system switches from one mode to another, i.e. when $x(k-1) \in \Omega_{j}$ and $x(k) \in \Omega_{i}$ for some $(i, j) \in \mathcal{S} \times \mathcal{S}, i \neq j$. Furthermore, observe that the two additional variables allow two types of non-monotone behavior of the tdCLF: $\lambda$ allows non-monotonicity of the tdCLF for fixed $k \in \mathbb{Z}_{+}$, while $\zeta$ allows nonmonotonicity of the tdCLF for fixed $x(k)$. The solution based on $\lambda$ was also used in 14] to stabilize hybrid systems with discrete dynamics (e.g., with discrete states and/or inputs) via non-monotone time-invariant CLFs.

In the remainder of the paper, for simplicity of exposition, we no longer consider non-monotone tdCLFs. However, all the derivations presented in the next section for Problem 1 trivially apply also to the case when (7b)-(7c) are replaced by (9a)-(9b), as $\lambda$ and $\zeta$, respectively, enter the latter inequalities linearly.

$\overline{{ }^{2} J(\cdot, \cdot): \mathbb{R}_{+}} \times \mathbb{R}_{+} \rightarrow \mathbb{R}_{+}$is radially unbounded and $J(0,0)=0$. 


\section{Synthesis of tdCLFs by a Single Linear Program}

In this section we consider candidate tvCLFs of a fixed structure and with a set of unknown parameters to be determined on-line, which yields a tdCLF. More precisely, we restrict our attention to cases where $V(k, x(k))=V(p(k), x(k))$, $V(\cdot, \cdot)$ is a priori defined and $p(k)$ is a vector of parameters. For example $V(k, x(k))=\|P(k) x(k)\|$ or $V(k, x(k))=x^{\top}(k) P(k) x(k)$, where in both cases the elements of $P(k)$ are the unknown parameters which are to be determined on-line so that (6)-(7) hold. Furthermore, to make solving Problem 1 on-line tractable, it is desirable that the inequalities (6)-(17) are convex in both the control input $u(k)$ and the parameters $p(k)$. In this respect we point out to some features of Problem 1 as follows. For a given $x(k)$, the lower bound inequality in (6) imposes convex constraints on the parameters $p(k)$ if and only if $V(p(k), x(k))$ is concave in $p(k)$, while the upper bound inequality in (6) imposes convex constraints on the parameters $p(k)$ if and only if $V(p(k), x(k))$ is convex in $p(k)$. Similarly, suppose that $\phi(x(k), u(k))$ and $x(k)$ are fixed. Then inequality (7b) imposes convex constraints on $p(k)$ if and only if $V(p(k), x(k))$ is both convex and concave in $p(k)$, i.e. if it is affine or linear in $p(k)$. To summarize, the inherent feature of Problem 1 is that, in general, it is a nonconvex problem.

In the remainder of this section we present a complete convexification procedure for the following fairly general case. In terms of the class of systems, we restrict our attention to $\mathrm{PWC}$ nonlinear systems that are affine in the control input, i.e.:

$$
\begin{aligned}
x(k+1)=\phi(x(k), u(k)) & =\phi_{j}(x(k), u(k)) \text { if } x(k) \in \Omega_{j} \\
& =f_{j}(x(k))+g_{j}(x(k)) u(k) \text { if } x(k) \in \Omega_{j}, \quad k \in \mathbb{Z}_{+}
\end{aligned}
$$

where $f_{j}(\cdot)$ and $g_{j}(\cdot)$ denote suitably defined continuous nonlinear functions. For brevity, let $f(x):=f_{j}(x)$ and $g(x):=g_{j}(x)$ if $x \in \Omega_{j}$, respectively. Observe that PWA systems are a subclass of the above system. Also, we assume that the sets $\mathbb{X}$ and $\mathbb{U}$ are polyhedra. In terms of candidate tvCLFs, we restrict our attention to functions defined using the infinity norm, i.e.:

$$
V(k, x(k)):=\|P(k) x(k)\|_{\infty},
$$

where $P(k) \in \mathbb{R}^{p \times n}$ is to be computed on-line so that (6)-(7) hold.

\subsection{Construction of the Lower and Upper Bound}

For a fixed $x(k) \in \mathbb{X}$ let

$$
\mathcal{P}(x(k)):=\left\{y \in \mathbb{R}^{n} \mid\langle y, x(k)\rangle \geq 0\right\},
$$

and let $\mathcal{P}_{i}(x(k)) \subset \mathbb{R}^{n}$ for $i \in\{1, \ldots, p\}=: \mathcal{I}, p \geq n$, be compact sets. Furthermore, define

$$
\Pi(x(k)):=\left\{P \in \mathbb{R}^{p \times n} \mid[P]_{i \bullet} \in \mathcal{P}_{i}(x(k)), \forall i \in \mathcal{I}\right\}
$$


and suppose that the collection of sets $\left\{\mathcal{P}_{i}(x(k))\right\}_{i \in \mathcal{I}}$ is such that:

$$
\begin{aligned}
& \mathcal{P}_{i}(x(k)) \subset \mathcal{P}(x(k)), \quad \forall i \in \mathcal{I}, \\
& P(k) \in \Pi(x(k)) \Rightarrow \quad \operatorname{rank}(P(k))=n, \\
& \mathcal{P}_{i}(x(k)) \cap \mathcal{B}_{r_{1}(k)}=\emptyset, \quad \mathcal{P}_{i}(x(k)) \subset \mathcal{B}_{r_{2}(k)}, \quad \forall i \in \mathcal{I},
\end{aligned}
$$

where $\mathcal{B}_{r_{i}(k)}:=\left\{z \in \mathbb{R}^{n} \mid\|z\|_{2}<r_{i}(k)\right\}, i=1,2$, for some $r_{1}(k), r_{2}(k) \in \mathbb{R}_{>0}$, $r_{1}(k)<r_{2}(k)$ for all $k \in \mathbb{Z}_{+}$.

Lemma 1. Let $x(k) \in \mathbb{X}, k \in \mathbb{Z}_{+}$, be fixed and let $\left\{\mathcal{P}_{i}(x(k))\right\}_{i \in \mathcal{I}}$ satisfy (12). Then

(i) $P(k) x(k) \geq 0$ for all $P(k) \in \Pi(x(k))$;

(ii) $\exists \alpha_{1} \in \mathcal{K}_{\infty}$ such that $\|P(k) z\|_{\infty} \geq \alpha_{1}\left(\|z\|_{\infty}\right), \forall z \in \mathbb{R}^{n}, \forall P(k) \in \Pi(x(k))$;

(iii) $\exists \alpha_{2} \in \mathcal{K}_{\infty}$ such that $\|P(k) z\|_{\infty} \leq \alpha_{2}\left(\|z\|_{\infty}\right), \forall z \in \mathbb{R}^{n}, \forall P(k) \in \Pi(x(k))$.

Proof. ( $i$ ) Follows directly from (12a) and the definitions of $\mathcal{P}(x(k))$ and $\Pi(x(k))$. (ii) Let

$$
c(k):=\max _{i \in \mathcal{I}} \min _{z \neq 0} \min _{y \in \mathcal{P}_{i}(x(k))} \frac{|\langle y, z\rangle|}{\|y\|_{2}\|z\|_{2}} .
$$

Note that $c(k)$ is well defined, as from (12c) we have that for each $i \in \mathcal{I}, y \in$ $\mathcal{P}_{i}(x(k)) \Rightarrow y \not \equiv 0$. For any $z \in \mathbb{R}^{n} \backslash\{0\}, \max _{i \in \mathcal{I}} \min _{y \in \mathcal{P}_{i}(x(k))} \frac{|\langle y, z\rangle|}{\|y\|_{2}\|z\|_{2}}>0$, since from (12b) it follows that there always exists a $j \in \mathcal{I}$ such that $y \in \mathcal{P}_{j}(x(k)) \Rightarrow$ $\langle y, z\rangle \neq 0$. Hence, $c(k) \neq 0$ and thus, $c(k)>0$. Now, let $P(k) \in \Pi(x(k))$ and for notational convenience let $p_{i}(k):=[P(k)]_{i \bullet}^{\top}$. Then we can write the following sequence of equalities

$$
\|P(k) z\|_{\infty}=\left\|\left(\left\langle p_{1}(k), z\right\rangle, \ldots,\left\langle p_{p}(k), z\right\rangle\right)^{\top}\right\|_{\infty}=\max _{i \in \mathcal{I}}\left|\left\langle p_{i}(k), z\right\rangle\right| .
$$

Furthermore, for any fixed $p_{i}(k)$ and any $z \neq 0$ we have

$$
\max _{i \in \mathcal{I}} \frac{\left|\left\langle p_{i}(k), z\right\rangle\right|}{\left\|p_{i}(k)\right\|_{2}\|z\|_{2}} \geq \max _{i \in \mathcal{I}} \min _{\tilde{z} \neq 0} \min _{\tilde{y} \in \mathcal{P}_{i}(x(k))} \frac{|\langle\tilde{y}, \tilde{z}\rangle|}{\|\tilde{y}\|_{2}\|\tilde{z}\|_{2}}=c(k) .
$$

Therefore, using (12c) and $\|z\|_{\infty} \leq\|z\|_{2}$, yields:

$$
\max _{i \in \mathcal{I}}\left|\left\langle p_{i}(k), z\right\rangle\right| \geq c(k)\left\|p_{i}(k)\right\|_{2}\|z\|_{2} \geq c(k) r_{1}(k)\|z\|_{\infty},
$$

which together with (14) implies $\|P(k) z\|_{\infty} \geq c(k) r_{1}(k)\|z\|_{\infty}$. Since $P(k)$ is an arbitrary matrix in $\Pi(x(k))$, we conclude that the desired inequality holds with $\alpha_{1}\left(\|z\|_{\infty}\right):=\inf _{k \in \mathbb{Z}_{+}} c(k) r_{1}(k)\|z\|_{\infty}$.

(iii) For any $P(k) \in \Pi(x(k))$ we have that

$$
\|P(k) z\|_{\infty} \leq\|P(k)\|_{\infty}\|z\|_{\infty}=\max _{i \in \mathcal{I}}\left\|p_{i}(k)\right\|_{1}\|z\|_{\infty}
$$

Using the fact that $\left\|p_{i}(k)\right\|_{1} \leq n\left\|p_{i}(k)\right\|_{2}$ and the property (12c), inequality (16) further implies that $\|P(k) z\|_{\infty} \leq n r_{2}(k)\|z\|_{\infty}$. Since $P(k)$ is an arbitrary matrix in $\Pi(x(k))$, we conclude that the desired inequality holds with $\alpha_{2}\left(\|z\|_{\infty}\right):=$ $n \sup _{k \in \mathbb{Z}_{+}} r_{2}(k)\|z\|_{\infty}$. 
Notice that the upper and lower bounds established in the proof of Lemma 1 can be derived explicitly as follows. Some tuning parameters $R_{1}, R_{2} \in \mathbb{R}_{>0}$ can always be a priori chosen such that $R_{1} \leq r_{1}(k)<r_{2}(k) \leq R_{2}$ for all $k \in \mathbb{Z}_{+}$. This will be illustrated in Section 4.3. where it is also shown how to derive a number $C \in \mathbb{R}_{>0}$ such that $c(k) \geq C$ for all $k \in \mathbb{Z}_{+}$. Furthermore, therein we present an approach to the derivation of the collection of sets $\left\{\mathcal{P}_{i}(x(k))\right\}_{i \in \mathcal{I}}$ such that (12) holds. Another clarifying point is that the result of Lemma 1 (i) will be instrumental in the convexification of inequality (7b).

\subsection{Convexification of Problem 1}

Next, let $D(x(k))$ and $d(x(k))$ be a matrix and a vector of appropriate dimensions such that at each time $k$ the inequalities (7a) are equivalently written as $D(x(k)) u(k) \leq d(x(k))$. Note that with the hypothesis that $\mathbb{X}$ and $\mathbb{U}$ are polyhedra, this can always be done for PWC nonlinear systems affine in control.

Problem 2. Let $C, R_{1}, R_{2} \in \mathbb{R}_{>0}$ with $R_{1}<R_{2}$ be given such that $C \leq c(k)$ and $R_{1} \leq r_{1}(k)<r_{2}(k) \leq R_{2}$ for all $k \in \mathbb{Z}_{+}$. At time $k \in \mathbb{Z}_{+}$let $x(k)$ be the measured state. Determine the partition $\left\{\mathcal{P}_{i}(x(k))\right\}_{i \in \mathcal{I}}, \mathcal{I}=\{1, \ldots, p\}, p \geq n$, such that (12) holds for some $r_{1}(k), r_{2}(k) \in \mathbb{R}_{>0}, r_{1}(k)<r_{2}(k)$. Then, compute $P(k) \in \mathbb{R}^{p \times n}, \tau(k) \in \mathbb{R}^{m}$ and $\xi(k) \in \mathbb{R}$ such that

$$
\begin{aligned}
& D(x(k)) \tau(k) \leq \xi(k) d(x(k)), \\
& \|\xi(k) f(x(k))+g(x(k)) \tau(k)\|_{\infty}-\rho[P(k) x(k)]_{i \bullet} \leq 0, \quad \forall i \in \mathcal{I}, \\
& \|P(k)\|_{\infty} \leq \xi(k), \\
& {[P(k)]_{i \bullet} \in \mathcal{P}_{i}(x(k)), \quad \forall i \in \mathcal{I},} \\
& \|P(k) x(k)\|_{\infty} \leq\|P(k-1) x(k)\|_{\infty}, \quad \text { if } \quad k \in \mathbb{Z}_{\geq 1} .
\end{aligned}
$$

Lemma 2. Let $P(k), \tau(k)$ and $\xi(k)$ denote a feasible solution of Problem 2 for state $x(k)$ at time $k \in \mathbb{Z}_{+}$and let $[u(k)]_{i}:=\frac{[\tau(k)]_{i}}{\xi(k)}$ for $i=1, \ldots, m$. Then $V(k, x):=\|P(k) x\|_{\infty}$ and $u(k)$ are a feasible solution of Problem 1 for state $x(k)$ at time $k \in \mathbb{Z}_{+}$.

Proof. Since (17d) implies that $P(k) \not \equiv 0$, we obtain $\|P(k)\|_{\infty} \neq 0$ and thus, from (17c) it follows that $\xi(k)>0$. This implies that $u(k)$ is indeed well-defined, and that we can pull out $\xi(k)$ from the norm in (17b). By Lemma 1f(i), we have that $(17 \mathrm{~d}) \Rightarrow P(k) x(k) \geq 0$. Furthermore, from with (17c) and (17b) we obtain:

$$
\begin{aligned}
0 & \geq \xi(k)\left\|f(x(k))+g(x(k)) \frac{\tau(k)}{\xi(k)}\right\|_{\infty}-\rho\|P(k) x(k)\|_{\infty} \\
& \geq\|P(k)\|_{\infty}\|f(x(k))+g(x(k)) u(k)\|_{\infty}-\rho\|P(k) x(k)\|_{\infty} \\
& \geq\|P(k)(f(x(k))+g(x(k)) u(k))\|_{\infty}-\rho\|P(k) x(k)\|_{\infty},
\end{aligned}
$$

i.e. (7b) holds. Furthermore, from (17a) we have that

$$
D(x(k)) \frac{\tau(k)}{\xi(k)}=D(x(k)) u(k) \leq d(x(k)),
$$


and therefore (7a) is satisfied. Using Lemma 1] the inequality (17d) and $R_{1} \leq$ $r_{1}(k)<r_{2}(k) \leq R_{2}$ for all $k \in \mathbb{Z}_{+}$we obtain (6) with $\alpha_{1}\left(\|x\|_{\infty}\right):=C R_{1}\|x\|_{\infty}$ and $\alpha_{2}\left(\|x\|_{\infty}\right):=n R_{2}\|x\|_{\infty}$. The proof is concluded by observing that (17e) is just (7c).

Remark 4. Suppose that each $\mathcal{P}_{i}(x(k))$ in Problem 2 is a convex set. Then Problem 2 amounts to finding a feasible solution to a set of convex inequalities, and it implicitly solves a non-convex optimization problem, i.e. Problem 1.

\subsection{Construction of a Collection of Polyehedral Sets $\left\{\mathcal{P}_{i}(x(k))\right\}_{i \in \mathcal{I}}$}

In parallel with the general description of the procedure we will refer to the following example for illustrative purposes. Suppose that $x \in \mathbb{R}^{2}$. For a fixed $x(k), k \in \mathbb{Z}_{+}$, Figure 2 illustrates a possible choice of the collection of sets $\left\{\mathcal{P}_{i}(x(k))\right\}_{i \in \mathcal{I}}$, where each set is a polyhedron. It is easy to verify that these sets satisfy the conditions (12). For example, by restricting the 3 non-zero vectors into the cones indicated by the angles $\varphi$ in Figure 2, it necessarily holds that at least two of these vectors are linearly independent. Hence, the full column rank condition $12 \mathrm{~b}$ is ensured.

Next we illustrate how to explicitly calculate the value of $c(k)$. Recall that $c(k):=\max _{i \in \mathcal{I}} \min _{z \neq 0} \min _{y \in \mathcal{P}_{i}(x(k))} \frac{|\langle y, z\rangle|}{\|y\|_{2}\|z\|_{2}}$ and for any $x, y \in \mathbb{R}^{n}$ the value $\frac{|\langle x, y\rangle|}{\|x\|_{2}\|y\|_{2}}$ defines the angle $\beta$ between the two vectors [20]. More precisely $\beta=$ $\cos ^{-1}\left(\frac{|\langle x, y\rangle|}{\|x\|_{2}\|y\|_{2}}\right), 0 \leq \beta \leq \frac{\pi}{2}$. As such, the value $c(k)$ is in fact the maximum of the smallest possible $\cos \left(\beta_{i}\right)$, where $\beta_{i}$ denotes the angle between $z$ and $y \in \mathcal{P}_{i}$. For the example of partition in Figure 2, we have that $c(k)=\cos (\varphi)=\cos \left(\frac{\pi}{3}\right)=0.5$ for all $k \in \mathbb{Z}_{+}$and thus, $C$ can be taken equal to 0.5 .

Next, we briefly describe an algorithm for constructing the sets $\left\{\mathcal{P}_{i}(x(k))\right\}_{i \in \mathcal{I}}$ as polyhedra, which consists of an off-line part and a very simple on-line adjustment procedure.

Off-line part: Construct an initial collection of polyhedral sets $\left\{\mathcal{P}_{i}^{0}\right\}_{i \in \mathcal{I}}$ for an arbitrary fixed $x(k)=x^{0}$ (for example, in Figure 2 we have chosen $x^{0}:=(1,0)^{\top}$ ) such that (12) holds. Note that this is always possible. In particular observe that the condition (12b) is satisfied if and only if $p \geq n$ and there does not exist a hyperplane in $\mathbb{R}^{n}$ which contains the origin and intersects all the sets $\left\{\mathcal{P}_{i}^{0}\right\}_{i \in \mathcal{I}}$. Since each $\mathcal{P}_{i}^{0}$ is a polyhedron, there exist matrices $H_{i}^{0}$ and vectors $h_{i}^{0}$ such that $y \in \mathcal{P}_{i}^{0} \Leftrightarrow H_{i}^{0} y \leq h_{i}^{0}$ for all $i \in \mathcal{I}$ (for the example of Figure 2, $\mathcal{I}=\{1,2,3\}$ ).

On-line part: Let $x(k)$ be measured and let $\alpha(x(k))$ be the angle between $x(k)$ and $x^{0}$, see Figure 2 for a graphical illustration. Then construct $\left\{\mathcal{P}_{i}(x(k))\right\}_{i \in \mathcal{I}}$ as follows: $H_{i}(x(k)):=H_{i}^{0} M(\alpha(x(k)))$ and $h_{i}(x(k))=h_{i}^{0}$, where $y \in \mathcal{P}_{i}(x(k)) \Leftrightarrow$ $H_{i}(x(k)) y \leq h_{i}(x(k))$ for all $i \in \mathcal{I}$ and the matrix $M(\alpha)$ is a suitably defined rotational matrix [20], which can be chosen off-line. For the example of Figure 2, $M=\left(\begin{array}{cc}\cos (\alpha) & \sin (\alpha) \\ -\sin (\alpha) & \cos (\alpha)\end{array}\right)$.

Remark 5. The on-line part mentioned above can be completely removed by replacing the terms $\rho[P(k) x(k)]_{i \bullet}, i \in \mathcal{I}$, in (17b) with the corresponding lower 


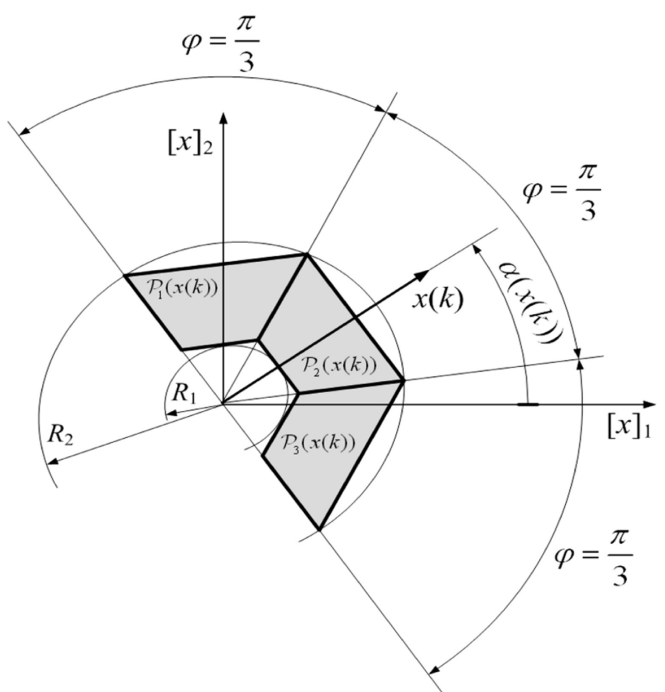

Fig. 2. Example of sets $\left\{\mathcal{P}_{i}(x(k))\right\}_{i \in \mathcal{I}}$ in the two dimensional case

bound. Also, observe that the derivation of $c(k)$ depends only on the off-line partition $\left\{\mathcal{P}_{i}^{0}\right\}_{i \in \mathcal{I}}$ and therefore, $c(k)=C$ can be fully determined off-line.

Note that now Problem 2 can be formulated as a single linear program as follows. Constraint (17a) is linear as $\mathbb{X}$ and $\mathbb{U}$ are polyhedra and the system is affine in the control input. Constraint (17d) is now a set of linear constraints as each set $\mathcal{P}_{i}(x(k))$ is a polyhedron for the measured state $x(k)$. Furthermore, note that that for any matrix $Z \in \mathbb{R}^{r \times l}$ the condition $\|Z\|_{\infty} \leq c$ for some $c \in \mathbb{R}_{>0}$ is equivalent to $\pm[Z]_{i 1} \pm[Z]_{i 2} \ldots \pm[Z]_{i l} \leq c, i=1, \ldots, r$. Thus, as $x(k)$ is known at each $k \in \mathbb{Z}_{+},(17 \mathrm{~b}),(17 \mathrm{c})$ and (17e) can be specified through a finite number of linear inequalities in $\xi(k), \tau(k)$ and in the elements of $P(k)$ without introducing any conservatism. Therefore, by Lemma 2, a solution to Problem 1 can be found by solving a single linear program at each sampling instant $k \in \mathbb{Z}_{+}$.

Remark 6 . The hybrid nature of the system dynamics is inherently embedded in inequality (17b), which is equivalent to

$$
\left\|\xi(k) f_{j}(x(k))+g_{j}(x(k)) \tau(k)\right\|_{\infty}-\rho[P(k) x(k)]_{i \bullet} \leq 0, \quad \forall i \in \mathcal{I}, \quad \text { if } x(k) \in \Omega_{j} .
$$

However, as $x(k)$ is known at every time instant $k \in \mathbb{Z}_{+}$, it implies that the index $j \in \mathcal{S}$ is also known. That is why it is possible to solve Problem 2 by a single linear program (LP). Moreover, even in the case of mode uncertainty (possibly due to measurement noise), one can impose the above inequality in a robust way, i.e. for all dynamics indexed by $j \in \overline{\mathcal{S}}(x(k)) \subseteq \mathcal{S}$, where $\overline{\mathcal{S}}(x(k))$ collects all the indexes corresponding to the regions $\Omega_{j}$ that need to be accounted for in the case of mode uncertainty. Then, Problem 2 still can be formulated as a single linear program, while the method presented in Section 3.1 can be employed to decrease conservativeness considerably. 

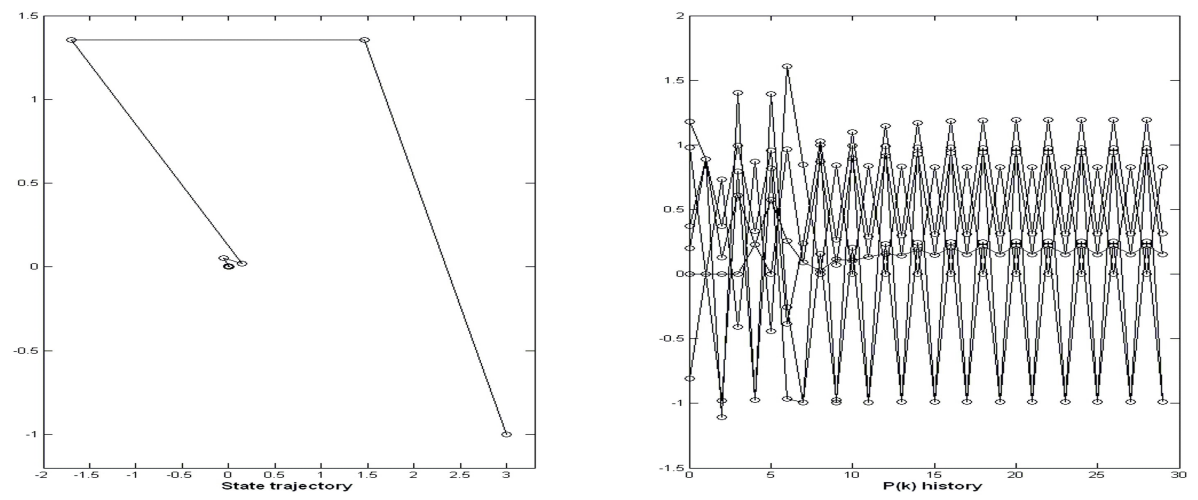

Fig. 3. Closed-loop simulation results: State-trajectory and $P(k)$ history

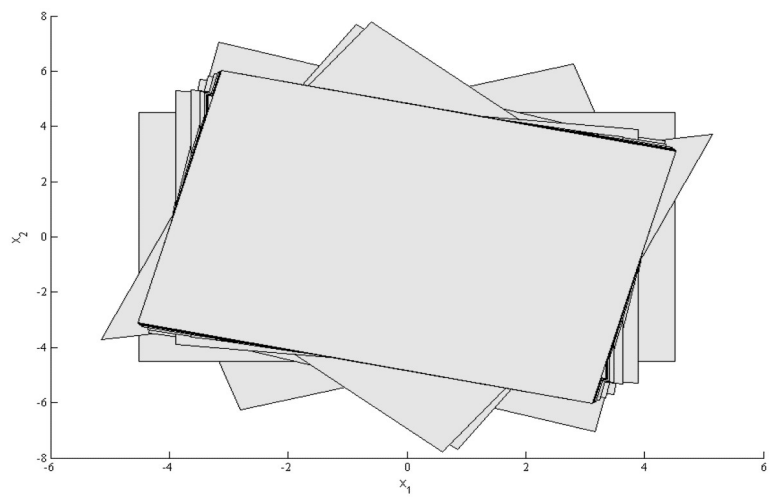

Fig. 4. Sublevel sets $\{x \mid V(k, x) \leq 4\}$ for $k \in \mathbb{Z}_{[0,30]}$

\section{$5 \quad$ Illustrative Example}

Consider system (11) with all the numerical details presented in Section 1.1 As stated therein, the synthesis problem for a PWQ Lyapunov function and a PWL state-feedback control law is not feasible unless the $\mathcal{S}$-procedure [10] is applied. This makes the synthesis of a stabilizing control law a challenging problem. We have followed the procedure described in the previous section to formulate Problem 2 as a single LP. We have fixed the dimensions of $P(k)$ to $\mathbb{R}^{3 \times 2}$. The initial partition $\left\{\mathcal{P}_{i}^{0}\right\}_{i \in \mathcal{I}}$ has been chosen as illustrated in Figure 2, which yields $c(k)=C=0.5$ for all $k \in \mathbb{Z}_{+}$. Furthermore, we have chosen the constants $R_{1}=1$ and $R_{2}=2$. The rotational matrix $M$ for finding the matrices $H_{i}(x(k))$, $i=1,2,3$, on-line is also taken as specified in Section 4.3. Finally, the tuning parameter $\rho$ is taken equal to 0.9 . The resulting LP has 8 optimization variables (the 6 elements of $P(k), \xi(k)$ and $\tau(k))$ and 44 constraints. The time spent to calculate the matrices $H_{i}(x(k))$, which update certain constraints in the LP, is 
negligible compared to the time required to solve the LP. The overall worst-case time required by the algorithm was less than 5 milliseconds.

Figure 3 shows the closed-loop simulation results for initial state $x(0)=$ $\left[\begin{array}{ll}3 & -1\end{array}\right]^{\top}$. The proposed method successfully stabilizes the PWL system, while satisfying state and input constraints $\left(\mathbb{X}:=\left\{x \in \mathbb{R}^{2} \mid\|x\|_{\infty} \leq 5\right\}\right.$ and $\mathbb{U}:=$ $\{u \in \mathbb{R}|| u \mid \leq 2\})$. Figure 4 presents a plot of the sublevel sets $\{x \mid V(k, x)=$ $\left.\|P(k) x\|_{\infty} \leq 4\right\}$ for $k \in \mathbb{Z}_{[0,30]}$. It is worth to point out that the closed-loop trajectory keeps on switching between two modes even very close to the origin, which in turn yields a different matrix $P(k)$ at two successive sampling instants. This can be observed in Figure 3, in the plot showing the history of all the 6 elements of $P(k)$, which still switch between two different values even when the state is very close to the origin. This demonstrates that the theoretical set-up proposed in this paper can effectively deal with non-trivial stabilization problems encountered in hybrid systems.

\section{Conclusions}

In this article we have proposed a new methodology that reduces significantly the conservatism of CLF design for discrete-time systems. Rather than searching for global CLFs off-line, we focused on synthesizing time-variant CLFs by solving on-line an optimization problem. As such, trajectory-dependent CLFs that are allowed to be locally non-monotone were derived. This approach offers a less conservative relaxation when compared to the classical $\mathcal{S}$-procedure approach. Regarding efficiency, we indicated that for PWC nonlinear systems affine in control and CLFs based on infinity norms, the on-line optimization problem can be formulated as a single linear program.

Acknowledgements. Research supported by the Veni grant "Flexible Lyapunov Functions for Real-time Control", grant number 10230, awarded by STW (Dutch Science Foundation) and NWO (The Netherlands Organization for Scientific Research).

\section{References}

1. Artstein, Z.: Stabilization with relaxed controls. Nonlinear Analysis 7, 1163-1173 (1983)

2. Sontag, E.D.: A Lyapunov-like characterization of asymptotic controllability. SIAM Journal of Control and Optimization 21, 462-471 (1983)

3. Sontag, E.D.: Stability and stabilization: Discontinuities and the effect of disturbances. In: Clarke, F.H., Stern, R.J. (eds.) Nonlinear Analysis, Differential Equations, and Control, pp. 551-598. Kluwer Academic Publishers, Dordrecht (1999)

4. Kokotović, P., Arcak, M.: Constructive nonlinear control: a historical perspective. Automatica 37(5), 637-662 (2001)

5. Grüne, L., Nesić, D.: Optimization based stabilization of sampled-data nonlinear systems via their approximate discrete-time models. SIAM Journal of Control and Optimization 42(1), 98-122 (2003) 
6. Kellett, C.M., Teel, A.R.: Discrete-time asymptotic controllability implies smooth control-Lyapunov function. Systems \& Control Letters 52, 349-359 (2004)

7. Jiang, Z.-P., Wang, Y.: Input-to-state stability for discrete-time nonlinear systems. Automatica 37, 857-869 (2001)

8. Lazar, M.: Model predictive control of hybrid systems: Stability and robustness. PhD thesis, Eindhoven University of Technology, The Netherlands (2006)

9. Branicky, M.S., Borkar, V.S., Mitter, S.K.: A unified framework for hybrid control: model and optimal control theory. IEEE Transactions on Automatic Control 43(1), 31-45 (1998)

10. Johansson, M., Rantzer, A.: Computation of piecewise quadratic Lyapunov functions for hybrid systems. IEEE Transactions on Automatic Control 43(4), 555-559 (1998)

11. Johansson, M.: Piecewise linear control systems. PhD thesis, Lund Institute of Technology, Sweden (1999)

12. Ferrari-Trecate, G., Cuzzola, F.A., Mignone, D., Morari, M.: Analysis of discretetime piecewise affine and hybrid systems. Automatica 38(12), 2139-2146 (2002)

13. Daafouz, J., Riedinger, P., Iung, C.: Stability analysis and control synthesis for switched systems: A switched Lyapunov function approach. IEEE Transactions on Automatic Control 47, 1883-1887 (2002)

14. Di Cairano, S., Lazar, M., Bemporad, A., Heemels, W.P.M.H.: A Control Lyapunov Approach to Predictive Control of Hybrid Systems. In: Egerstedt, M., Mishra, B. (eds.) HSCC 2008. LNCS, vol. 4981, pp. 130-143. Springer, Heidelberg (2008)

15. Grieder, P., Kvasnica, M., Baotic, M., Morari, M.: Stabilizing low complexity feedback control of constrained piecewise affine systems. Automatica 41(10), 1683-1694 (2005)

16. Lazar, M., Heemels, W.P.M.H., Weiland, S., Bemporad, A.: Stabilizing model predictive control of hybrid systems. IEEE Transactions on Automatic Control 51(11), 1813-1818 (2006)

17. Jiang, Z.-P., Wang, Y.: A converse Lyapunov theorem for discrete-time systems with disturbances. Systems \& Control Letters 45, 49-58 (2002)

18. Kellett, C.M., Teel, A.R.: On the robustness of $\mathcal{K} \mathcal{L}$-stability for difference inclusions: Smooth discrete-time Lyapunov functions. SIAM Journal on Control and Optimization 44(3), 777-800 (2005)

19. Vorotnikov, V.I.: Partial stability and control. Birkhäuser, Boston (1998)

20. Horn, R.A., Johnson, C.R.: Matrix Analysis. Cambridge University Press, United Kingdom (1985) 